\title{
A Sliding Mode Controller for Under-actuated VTOL Aircraft
}

\author{
Yang Zhao \\ Department of Mechanical and Electrical Engineering, \\ Guangdong University of Science\&Technology \\ Dongguan523083, China \\ E-mail:zhaoyang19781023@gmail.com
}

\begin{abstract}
The trajectory tracking control for the under-actuated VTOL (vertical take off and landing) aircraft is addressed. VTOL aircraft is an under-actuated system with three degrees of freedom and two control inputs. The motion equations of the system are transformed into underactuated standard form using coordinate transformations and the Sliding Mode Controller is designed. Then, the Lyapunov stability analysis shows that the control strategy guarantees the stability of the closed-loop system.Simulation results indicate that the proposed control scheme can make VTOL aircraft track the desired trajectory rapidly.
\end{abstract}

Keywords: VTOL aircraft, sliding mode control, under-actuated, trajectory tracking

\section{Introduction}

Vertical takeoff and landing (VTOL) aircraft could achieve free takeoff and landing without being limited by runway, so it has important military value ${ }^{[1]}$. VTOL aircraft system is under-actuated, non-minimum-phase and non-linear. As it is very challenging, its attitude control has aroused extensive attention of scholars at home and abroad. Typically, due to the decreasing number of input stimulus in the under-actuated system, some state variables in the configuration space of the system have no corresponding control inputs, namely, some degree of freedom of the system is under-actuated. This feature leads to the fact that such systems often have some non holonomic constraint equations ${ }^{[2]}$, therefore, the state variables of the system locate on an indeterminate configuration, which greatly increases the difficulty of the control design of this kind of system.

Due to the improving requirements for anti-interference ability and robust performance of under-actuated system, the sliding mode variable structure control method has been gradually applied. For the under-actuated mechanical system, its status variables fail to achieve decoupling, which leads to the fact that the sliding mode variable structure control method cannot be directly applied to such systems. To solve this problem, Orlov et al adopted the sliding mode control to specify a critical state, then the switches could switch back and forth indefinitely and the system could swing around the target point. This has overcome some uncertain difficulties and outside interference to a certain extent ${ }^{[3]}$. Some domestic scholars also proposed some improved sliding mode variable structure control methods based on the classical one ${ }^{[4,5]}$, and the representative solution is the double sliding mode control method ${ }^{[6,7]}$ and cascaded sliding mode control method ${ }^{[8]}$. The former means to design a sliding surface for each subsystem of the under-actuated system and construct a sliding mode surface for the whole system based on the previous ones. On this basis, seek control law to make the sliding surface of each subsystem tends to zero, so that the state variables of the entire system approach zero. The latter means to design a sliding mode surface for the first subsystem and then design the second one based on the previous one and the next state variable, and so on. The final sliding mode surface 
contains information of all state variables, and as long as the sliding mode surface tends to zero, all of the other surfaces tend to zero, so do the state variables. Compared with other control methods, the biggest advantage of using sliding mode variable structure control method lies in its strong robustness which could be used to directly handle the strong nonlinear flight control system. However, this control method requires switching between different switching surfaces, which will inevitably cause buffeting problems, so that the energy consumption increases. Therefore, how to overcome this disadvantage to achieve the superior performance of sliding mode variable structure control method is the current research focus.

\section{Mathematical Model of the Controlled Object}

Figure 1 is the free-body diagram of VTOL aircraft on $O x y$ plane, which considers the vertical $y$-axis and lateral $\mathrm{x}$-axis and ignores the exercise in front and back (ie, $\mathrm{z}$ direction). $O x y$ is the inertial coordinate system.



\section{Figure 1. The Free-body Diagram of VTOL Aircraft}

The mechanism analysis method is used to establish dynamic equilibrium equation of VTOL aircraft ${ }^{[9]}$.

$$
\left\{\begin{array}{l}
\ddot{x}=-u_{1} \sin \theta+\varepsilon u_{2} \cos \theta \\
\ddot{y}=u_{1} \cos \theta+\varepsilon u_{2} \sin \theta-g \\
\ddot{\theta}=u_{2}
\end{array}\right.
$$

In the equation, $x$ and $y$ represent the location of the centroid of the aircraft, $\theta$ is the rolling angle of the aircraft; $u_{1}$ and $u_{2}$ are the control input, that is, the bottom thrust and roll moment of the aircraft; $\mathrm{g}$ is the gravitational acceleration; $\varepsilon$ is the coefficient to describe the coupling relationship between $u_{1}$ and $u_{2}$, if $\varepsilon<0$, apply an aligning moment to scroll it to the left, thus generating a rightward accelerated speed. Conversely, a leftward accelerated speed is generated.

Equation (1) indicates that there is coupling in parameters of this model and it is also a typical under-actuated system, that is, it has three degrees of freedom $x, y$ and $\theta$, two control inputs $u_{1}$ and $u_{2}$. The number of input is smaller than that of the output, so the arbitrary trajectory tracking cannot be conducted for three output of the system. 


\section{Decoupling of Parameters of the Controlled Object}

Make $x_{1}=x, x_{2}=\dot{x}, y_{1}=y, y_{2}=\dot{y}, \omega=\dot{\theta}$, the equation (1) can be converted to equation (2).

$$
\left\{\begin{array}{l}
\dot{x}_{1}=x_{2} \\
\dot{x}_{2}=-u_{1} \sin \theta+\varepsilon u_{2} \cos \theta \\
\dot{y}_{1}=y_{2} \\
\dot{y}_{2}=u_{1} \cos \theta+\varepsilon u_{2} \sin \theta-g \\
\dot{\theta}=\omega \\
\dot{\omega}=u_{2}
\end{array}\right.
$$

Conduct decoupling operation for equation (2) by four steps

(1) The first decoupling: the elimination of coupling of $\dot{x}_{2}$ and $\dot{y}_{2}$. By the equation (2), the following equation can be obtained:

Design the decoupling parts:

$$
\left[\begin{array}{l}
\dot{x}_{1} \\
\dot{y}_{1}
\end{array}\right]=\left[\begin{array}{cc}
-\sin \theta & \varepsilon \cos \theta \\
\cos \theta & \varepsilon \sin \theta
\end{array}\right]\left[\begin{array}{l}
u_{1} \\
u_{2}
\end{array}\right]+\left[\begin{array}{c}
0 \\
-g
\end{array}\right]
$$

$$
\left[\begin{array}{l}
u_{1} \\
u_{2}
\end{array}\right]=\left[\begin{array}{cc}
-\sin \theta & \varepsilon \cos \theta \\
\cos \theta & \varepsilon \sin \theta
\end{array}\right]^{-1}\left[\begin{array}{c}
v_{\mathrm{m} 1} \\
v_{\mathrm{m} 2}+g
\end{array}\right]
$$

In equation (3), $v_{\mathrm{m} 1}$ and $v_{\mathrm{m} 2}$ are the control terms to be designed, and the equation (2) is converted to equation (4)

$$
\begin{aligned}
& \left\{\begin{array}{l}
\dot{x}_{1}=v_{\mathrm{m} 1} \\
\dot{y}_{1}=v_{\mathrm{m} 2} \\
\ddot{\theta}=u_{2}
\end{array}\right. \\
& {\left[\begin{array}{cc}
-\sin \theta & \varepsilon \cos \theta \\
\cos \theta & \varepsilon \sin \theta
\end{array}\right]^{-1}=\left[\begin{array}{cc}
-\sin \theta & \cos \theta \\
\frac{1}{\varepsilon} \cos \theta & \frac{1}{\varepsilon} \sin \theta
\end{array}\right]} \\
& \ddot{\theta}=u_{2}=\frac{1}{\varepsilon} \cos \theta\left[v_{\mathrm{m} 1}+\frac{1}{\varepsilon} \sin \theta\left(v_{\mathrm{m} 2}+g\right)\right.
\end{aligned}
$$

Therefore, the equation (4) is converted to the equation (5).

$$
\left\{\begin{aligned}
\dot{x}_{1} & =v_{\mathrm{m} 1} \\
\dot{y}_{1} & =v_{\mathrm{m} 2} \\
\varepsilon \ddot{\theta} & =\cos \theta \square v_{\mathrm{m} 1}+\sin \theta \llbracket\left(v_{\mathrm{m} 2}+g\right)
\end{aligned}\right.
$$

(2) The second decoupling: the elimination of the coupling of $v_{\mathrm{m} 1}$ and $v_{\mathrm{m} 2}$ in $\varepsilon \ddot{\theta}$ [10]. Make:

$$
\begin{gathered}
x_{c}=x_{1}-\varepsilon \sin \theta \\
y_{c}=y_{1}-\varepsilon \cos \theta \\
v_{\mathrm{m} 1}=\left(v_{1}-\varepsilon \dot{\theta}\right) \sin \theta+\varepsilon v_{2} \cos \theta \\
v_{\mathrm{m} 2}=-\left(v_{1}-\varepsilon \dot{\theta}\right) \cos \theta+\varepsilon v_{2} \sin \theta-g
\end{gathered}
$$


$x_{c}$ and $y_{c}$ are the output of conversion, $v_{1}$ and $v_{2}$ are the input after conversion. Put equation (8) and (9) in $\varepsilon \ddot{\theta}$ of equation (5) and obtain:

$$
\begin{aligned}
\varepsilon \ddot{\theta}= & \cos \theta\left(\left(v_{1}-\varepsilon \dot{\theta}\right) \sin \theta+\varepsilon v_{2} \cos \theta\right)+\sin \theta \\
& \left(\left(-\left(v_{1}-\varepsilon \dot{\theta}\right) \cos \theta+\varepsilon v_{2} \sin \theta-g\right)+g\right) \\
= & \varepsilon v_{2}(\cos \theta)^{2}+\varepsilon v_{2}(\sin \theta)^{2} \\
= & \varepsilon v_{2}
\end{aligned}
$$

Take the second-order derivative for equation (6) and put equation (8) and (9) in it:

$$
\begin{aligned}
\ddot{x}_{c} & =\ddot{x}_{1}-\left(-\varepsilon \sin \theta \square \dot{\theta}^{2}+\varepsilon \cos \theta \sqsubset \ddot{\theta}\right) \\
& =\left(v_{1}-\varepsilon \dot{\theta}\right) \sin \theta+\varepsilon v_{2} \cos \theta \\
& =v_{1} \sin \theta
\end{aligned}
$$

Take the second-order derivative for equation (7) and put equation (8) and (9) in it:

$$
\begin{aligned}
& \ddot{y}_{c}=\ddot{y}_{1}+\left(-\varepsilon \cos \theta\left[\dot{\theta}^{2}-\varepsilon \sin \theta \ddot{\theta}\right)\right.
\end{aligned}
$$

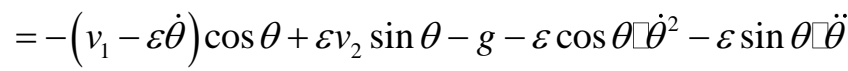

$$
\begin{aligned}
& =-v_{1} \cos \theta-g
\end{aligned}
$$

Constitute a new systematic equation by equation (10), (11) and (12):

$$
\left\{\begin{array}{l}
\ddot{x}_{c}=v_{1} \sin \theta \\
\ddot{y}_{c}=-v_{1} \cos \theta-g \\
\ddot{\theta}=v_{2}
\end{array}\right.
$$

(3) The third decoupling: the elimination the coupling of $\sin \theta$ and $\cos \theta$. Make:

$$
\begin{gathered}
h_{1}=v_{1} \cos \theta \\
v_{1}=\frac{h_{1}}{\cos \theta} \\
\ddot{p}_{1}=\ddot{x}_{c}=v_{1} \sin \theta=\frac{h_{1}}{\cos \theta} \sin \theta=h_{1} \tan \theta \\
\ddot{p}_{2}=\ddot{y}_{c}=-h_{1}-g
\end{gathered}
$$

Make $p_{3}=\tan \theta$, and:

$$
\begin{aligned}
\ddot{p}_{3} & =\left(\frac{\sin \theta}{\cos \theta}\right)^{\prime \prime}=\left(\frac{\dot{\theta}}{\cos ^{2} \theta}\right)^{\prime} \\
& =\frac{\ddot{\theta} \cos ^{2} \theta+2 \dot{\theta} \cos ^{3} \theta \sin \theta}{\cos ^{4} \theta} \\
& =\frac{v_{2} \cos ^{2} \theta+2 \dot{\theta} \cos ^{3} \theta \sin \theta}{\cos ^{4} \theta}
\end{aligned}
$$

Make $h_{2}=\ddot{p}_{3}$, and equation (13) is converted to equation (16).

$$
\left\{\begin{array}{l}
\ddot{p}_{1}=p_{3} h_{1} \\
\ddot{p}_{2}=-h_{1}-g \\
\ddot{p}_{3}=h_{2}
\end{array}\right.
$$


$h_{1}$ and $h_{2}$ are the control inputs after conversion. The relation between $h_{1}$ and $v_{1}$ is as shown in equation (14), and the relation between $h_{2}$ and $v_{2}$ is obtained by the equation (15).

$$
h_{2}=\frac{v_{2}+2 \dot{\theta}^{2} \tan \theta}{\cos ^{2} \theta}
$$

Make $q_{1}=p_{1}, q_{2}=\dot{p}_{1}, q_{3}=p_{2}, q_{4}=\dot{p}_{2}, q_{5}=p_{3}, q_{6}=\dot{p}_{3}$, and equation (16) is converted to equation (17).

$$
\left\{\begin{array}{l}
\dot{q}_{1}=q_{2} \\
\dot{q}_{2}=q_{5} h_{1} \\
\dot{q}_{3}=q_{4} \\
\dot{q}_{4}=-h_{1}-g \\
\dot{q}_{5}=q_{6} \\
\dot{q}_{6}=h_{2}
\end{array}\right.
$$

(4) The fourth decoupling: the conversion is the under-actuated standard form. The equation (17) is converted to equation (18).

$$
\left\{\begin{array}{l}
\dot{z}_{1}=z_{2} \\
\dot{z}_{2}=f_{1}\left(z_{1}, z_{2}, z_{3}, z_{4}\right) \\
\dot{z}_{3}=z_{4} \\
\dot{z}_{4}=f_{2}\left(z_{1}, z_{2}, z_{3}, z_{4}\right)+\boldsymbol{b h}
\end{array}\right.
$$

In the above equation,

$$
\begin{gathered}
\boldsymbol{z}_{1}=\left[\begin{array}{l}
q_{1}+\int_{0}^{t} q_{4} q_{5} \mathrm{~d} t \\
\left.\int_{0}^{t}\left(\int_{0}^{t} q_{3} \mathrm{~d} t\right) \mathrm{d} t\right]
\end{array}\right] \\
\boldsymbol{z}_{2}=\left[\begin{array}{c}
q_{2}+q_{4} q_{5} \\
\int_{0}^{t} q_{3} \mathrm{~d} t
\end{array}\right] \\
\boldsymbol{z}_{3}=\left[\begin{array}{c}
z_{3}(1) \\
z_{3}(2)
\end{array}\right]=\left[\begin{array}{l}
q_{3} \\
q_{5}
\end{array}\right] \\
\boldsymbol{z}_{4}=\left[\begin{array}{c}
z_{4}(1) \\
z_{4}(2)
\end{array}\right]=\left[\begin{array}{l}
q_{4} \\
q_{6}
\end{array}\right] \\
f_{1}\left(\boldsymbol{z}_{1}, \boldsymbol{z}_{2}, \boldsymbol{z}_{3}, \boldsymbol{z}_{4}\right)=\left[\begin{array}{c}
-g q_{5}+q_{4} q_{6} \\
q_{3}
\end{array}\right] f_{2}\left(z_{1}, z_{2}, z_{3}, z_{4}\right)=\left[\begin{array}{c}
-g \\
0
\end{array}\right] \\
\boldsymbol{b}=\left[\begin{array}{cc}
-1 & 0 \\
0 & 1
\end{array}\right] \\
\boldsymbol{h}=\left[\begin{array}{l}
h_{1} \\
h_{2}
\end{array}\right]
\end{gathered}
$$

The equivalence analysis of equation (17) and (18) is shown as follows.

(1) $\dot{z}_{1}=z_{2}$ and obtain $\left[\begin{array}{c}\dot{q}_{1}+q_{4} q_{5} \\ \int_{0}^{t} q_{3} \mathrm{~d} t\end{array}\right]=\left[\begin{array}{c}q_{2}+q_{4} q_{5} \\ \int_{0}^{t} q_{3} \mathrm{~d} t\end{array}\right], \dot{q}_{1}=q_{2}$. 
(2) $\dot{z}_{2}=f_{1}\left(z_{1}, z_{2}, z_{3}, z_{4}\right)$ and obtain

$$
\left[\begin{array}{c}
\dot{q}_{2}+\dot{q}_{4} q_{5}+q_{4} \dot{q}_{5} \\
q_{3}
\end{array}\right]=\left[\begin{array}{c}
\dot{q}_{2}+\left(-h_{1}-g\right) q_{5}+q_{4} q_{6} \\
q_{3}
\end{array}\right]=\left[\begin{array}{c}
-g q_{5}+q_{4} q_{6} \\
q_{3}
\end{array}\right], \dot{q}_{2}=q_{5} h_{1} .
$$

(3) $\dot{z}_{3}=z_{4}$ and obtain $\left[\begin{array}{c}\dot{q}_{3} \\ \dot{q}_{5}\end{array}\right]=\left[\begin{array}{c}q_{4} \\ q_{6}\end{array}\right]$.

(4) $\dot{z}_{4}=f_{2}\left(z_{1}, z_{2}, z_{3}, z_{4}\right)+\boldsymbol{b h}$ and obtain $\left[\begin{array}{l}\dot{q}_{4} \\ \dot{q}_{6}\end{array}\right]=\left[\begin{array}{c}-h_{1}-g \\ h_{2}\end{array}\right]$.

From the above derivation, conduct equivalent transformation for equation (17) to the under-actuated standard form of equation (18) and design the controller according to this form.

\section{Design of the Sliding Mode Control Law}

Assume that the control law is shown as follows:

$$
\boldsymbol{h}=\boldsymbol{u}_{\mathrm{eq}}+\boldsymbol{u}_{\mathrm{sw}}
$$

In equation (19), $\boldsymbol{u}_{\mathrm{eq}}$ is the equivalent control term, $\boldsymbol{u}_{\mathrm{sw}}$ is the switching control term.For the equation (18), assume the tracking variable of $z_{1}$ is $z_{1 \mathrm{~d}}$ and then the error signal is shown as [11]:

$$
\begin{gathered}
\boldsymbol{e}_{1}=z_{1}-z_{1 \mathrm{~d}} \\
\boldsymbol{e}_{2}=\dot{\boldsymbol{e}}_{1}=z_{2}-\dot{z}_{1 \mathrm{~d}} \\
\boldsymbol{e}_{3}=\ddot{\boldsymbol{e}}_{1}=\dot{\boldsymbol{z}}_{2}-\ddot{z}_{1 \mathrm{~d}}=f_{1}-\ddot{z}_{1 \mathrm{~d}} \\
\boldsymbol{e}_{4}=\dddot{\boldsymbol{e}}_{1}=\dot{f}_{1}-\dddot{z}_{1 \mathrm{~d}} \\
=\frac{\partial f_{1}}{\partial \boldsymbol{z}_{1}} \boldsymbol{z}_{2}+\frac{\partial f_{1}}{\partial \boldsymbol{z}_{2}} f_{1}+\frac{\partial f_{1}}{\partial \boldsymbol{z}_{3}} \boldsymbol{z}_{4}-\dddot{\boldsymbol{z}}_{1 \mathrm{~d}} \\
\mathrm{As} \frac{\partial f_{1}}{\partial \boldsymbol{z}_{1}}=\frac{\partial f_{1}}{\partial \boldsymbol{z}_{2}}=0, \text { and: } \\
\boldsymbol{e}_{4}=\frac{\partial f_{1}}{\partial \boldsymbol{z}_{3}} \boldsymbol{z}_{4}-\dddot{z}_{\mathrm{ld}}
\end{gathered}
$$

Make the sliding mode surface is:

$$
\boldsymbol{s}=c_{1} \boldsymbol{e}_{1}+c_{2} \boldsymbol{e}_{2}+c_{3} \boldsymbol{e}_{3}+\boldsymbol{e}_{4}\left(c_{i}>0, i=1,2,3\right)
$$

Make $\dot{\boldsymbol{s}}=0$ and $\boldsymbol{h}=\boldsymbol{u}_{\mathrm{eq}}, c_{1} \dot{\boldsymbol{e}}_{1}+c_{2} \dot{\boldsymbol{e}}_{2}+c_{3} \dot{\boldsymbol{e}}_{3}+\dot{\boldsymbol{e}}_{4}=0$.

$$
c_{1}\left(z_{2}-\dot{z}_{1 \mathrm{~d}}\right)+c_{2}\left(f_{1}-\ddot{z}_{1 \mathrm{~d}}\right)+c_{3}\left(\frac{\partial f_{1}}{\partial \boldsymbol{z}_{3}} \boldsymbol{z}_{4}-\dddot{\boldsymbol{z}}_{1 \mathrm{~d}}\right)+\left(\left(\frac{\partial f_{1}}{\partial \boldsymbol{z}_{3}} z_{4}\right)^{\prime}-\dddot{z}_{1 \mathrm{~d}}\right)=0
$$

Put $\dot{\boldsymbol{z}}_{4}$ of equation (18) in equation (21), and $\boldsymbol{h}=\boldsymbol{u}_{\text {eq }}$ and obtain:

$$
c_{1}\left(z_{2}-\dot{z}_{1 \mathrm{~d}}\right)+c_{2}\left(f_{1}-\ddot{\boldsymbol{z}}_{1 \mathrm{~d}}\right)+c_{3}\left(\frac{\partial f_{1}}{\partial \boldsymbol{z}_{3}} \boldsymbol{z}_{4}-\dddot{\boldsymbol{z}}_{\mathrm{ld}}\right)+\left(\frac{\partial f_{1}}{\partial \boldsymbol{z}_{3}}\left(f_{2}+\boldsymbol{b} \boldsymbol{u}_{\mathrm{eq}}\right)-\dddot{\boldsymbol{z}}_{\mathrm{dd}}\right)=0
$$

Because 


$$
\frac{\partial f_{1}}{\partial z_{3}}=\left[\begin{array}{cc}
\frac{\partial\left(-g q_{5}+q_{4} q_{6}\right)}{\partial q_{3}} & \frac{\partial\left(-g q_{5}+q_{4} q_{6}\right)}{\partial q_{5}} \\
\frac{\partial f_{1}}{\partial q_{3}} & \frac{\partial f_{1}}{\partial q_{5}}
\end{array}\right]=\left[\begin{array}{cc}
0 & -g \\
1 & 0
\end{array}\right]
$$

Therefore $\frac{\partial f_{1}}{\partial z_{3}}$ is reversible, so the following equation can be obtained by the equation (22):

$$
\boldsymbol{u}_{\mathrm{eq}}=\left(\frac{\partial f_{1}}{\partial \boldsymbol{z}_{3}} \boldsymbol{b}\right)^{-1}\left(c_{1}\left(z_{2}-\dot{z}_{1 \mathrm{~d}}\right)+c_{2}\left(f_{1}-\ddot{z}_{\mathrm{ld}}\right)+c_{3}\left(\frac{\partial f_{1}}{\partial z_{3}} \boldsymbol{z}_{4}-\dddot{z}_{\mathrm{ld}}\right)+\left(\frac{\partial f_{1}}{\partial \boldsymbol{z}_{3}} f_{2}-\dddot{z}_{\mathrm{ld}}\right)\right)
$$

As $\dot{z}_{1}=z_{2}, \ddot{z}_{1}=f_{1}, \dddot{z}_{1}=\dot{f}_{1}=\frac{\partial f_{1}}{\partial z_{3}} z_{4}, \dddot{z}_{1}=\frac{\partial f_{1}}{\partial z_{3}} \dot{z}_{4}, \dot{z}_{1 \mathrm{~d}} \ddot{z}_{1 \mathrm{~d}} \dddot{z}_{1 \mathrm{~d}}$ and $\dddot{z}_{\mathrm{dd}}$ in equation (23) can be obtained.

Design the switching term control:

$$
\boldsymbol{u}_{\mathrm{sw}}=-\left(\frac{\partial f_{1}}{\partial \boldsymbol{z}_{3}} \boldsymbol{b}\right)^{-1}(M \operatorname{sgn}(s)-\lambda \boldsymbol{s})(M>0)
$$

In equation (24), $\operatorname{sgn}(s)$ can be taken as equation (25).

$$
\operatorname{sgn}\left(s_{i}\right)= \begin{cases}+1 & s_{i} \geq 0 \\ -1 & s_{i}<0\end{cases}
$$

The control law can be obtained from equation (19) (23) (24).

In equation (24), $\operatorname{sgn}(s)$ control strategy will increase the sliding mode buffeting, so in the actual application, the linear function of the boundary layer could be adopted:

$$
\operatorname{sgn}(s)=\left\{\begin{array}{cc}
+1 & s_{i}>\Delta \\
k s_{i} & \left|s_{i}\right| \leq \Delta \\
-1 & s_{i}<-\Delta
\end{array}\right.
$$

\section{Analysis of Control System Stability}

Put equation (23) and (24) in equation (19) and then plug $\dot{s}$ into it.

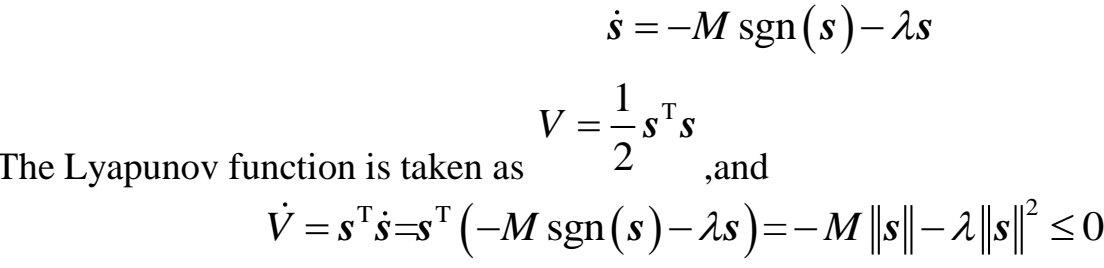

Therefore, the controller equation (19) could ensure the accessibility of sliding mode surface. When it reaches the sliding mode surface, $s=0$, the following equation can be obtained.

$$
\boldsymbol{e}_{4}=-c_{1} \boldsymbol{e}_{1}-c_{2} \boldsymbol{e}_{2}-c_{3} \boldsymbol{e}_{3}
$$


$\boldsymbol{E}_{1}=\left[\begin{array}{lll}\boldsymbol{e}_{1} & \boldsymbol{e}_{2} & \boldsymbol{e}_{3}\end{array}\right]^{\mathrm{T}}$ and the error state equation can be written as:

$$
\boldsymbol{A}=\left[\begin{array}{ccc}
0 & 1 & 0 \\
0 & 0 & 1 \\
-c_{1} & -c_{2} & -c_{3}
\end{array}\right], \boldsymbol{A} \text { could meet the matrix condition of hurwitz. Take }
$$

$$
\dot{\boldsymbol{E}}_{1}=\boldsymbol{A} \boldsymbol{E}_{1}
$$

For equation (29), the Lyapunov function is taken as

$$
\begin{aligned}
V_{1} & =\boldsymbol{E}_{1}^{\mathrm{T}} \boldsymbol{P} \boldsymbol{E}_{1}\left(\boldsymbol{P}=\boldsymbol{P}^{\mathrm{T}}>0\right) \\
\dot{V}_{1} & =\dot{\boldsymbol{E}}_{1}^{\mathrm{T}} \boldsymbol{P} \boldsymbol{E}_{1}+\boldsymbol{E}_{1}^{\mathrm{T}} \boldsymbol{P} \dot{\boldsymbol{E}}_{1} \\
& =\left(A \boldsymbol{E}_{1}\right)^{\mathrm{T}} \boldsymbol{P} \boldsymbol{E}_{1}+\boldsymbol{E}_{1}^{\mathrm{T}} \boldsymbol{P}\left(A \boldsymbol{E}_{1}\right) \\
& =\boldsymbol{E}_{1}^{\mathrm{T}} A^{\mathrm{T}} \boldsymbol{P} \boldsymbol{E}_{1}+\boldsymbol{E}_{1}^{\mathrm{T}} \boldsymbol{P} A \boldsymbol{E}_{1} \\
& =\boldsymbol{E}_{1}^{\mathrm{T}}\left(A^{\mathrm{T}} \boldsymbol{P}+\boldsymbol{P} A\right) \boldsymbol{E}_{1}
\end{aligned}
$$

As $\boldsymbol{A}$ is hurwitz matrix, there exists $Q_{\text {to meet Lyapunov equation: }}$

$$
A^{\mathrm{T}} \boldsymbol{P}+\boldsymbol{P} A=-\boldsymbol{Q}\left(\boldsymbol{Q}=\boldsymbol{Q}^{\mathrm{T}}>0\right)
$$

Put equation (31) into equation (30) and obtain equation (32)

$$
\dot{V}_{1}=\boldsymbol{E}_{1}^{\mathrm{T}} \boldsymbol{Q} \boldsymbol{E}_{1} \leq-\lambda_{\min }(\boldsymbol{Q})
$$

In the equation, $\lambda_{\min }(\boldsymbol{Q})$ is the smallest eigenvalue of the positively definite matrix $Q$.

Equation (32) $\dot{V}_{1} \leq 0$ shows that when $t \rightarrow \infty, \boldsymbol{e}_{1} \rightarrow 0, \boldsymbol{e}_{2} \rightarrow 0, \boldsymbol{e}_{3} \rightarrow 0$, $z_{1} \rightarrow z_{1 \mathrm{~d}}, z_{2} \rightarrow z_{2 \mathrm{~d}}, z_{3} \rightarrow z_{3 \mathrm{~d}}$. By equation (28), when $\boldsymbol{e}_{1} \boldsymbol{e}_{2}$ and $\boldsymbol{e}_{3}$ tend to 0 , $\boldsymbol{e}_{4} \rightarrow 0_{\mathrm{So}} z_{4} \rightarrow z_{4 \mathrm{~d}}, x \rightarrow x_{\mathrm{d}}, y \rightarrow y_{\mathrm{d}}, \theta \rightarrow \theta_{\mathrm{d}}$.

\section{Simulation Study}

For the controlled object equation (1), take $\varepsilon=10, g=9.8$ and predetermine the trajectory $x_{\mathrm{d}}=t \quad y_{\mathrm{d}}=\sin t \theta_{\mathrm{d}}=0$. Design the control law according to equation (19). In order to make $A$ become the hurwitz matrix, $c_{1}=27, c_{2}=27, c_{3}=9, M=0.1, \lambda=0.1$. The initial state of the controlled object is taken as $\left[\begin{array}{llllll}0.2 & 0 & 0.2 & 0 & 0.1 & 0\end{array}\right], \operatorname{sgn}\left(s_{i}\right)$ is taken according to equation (26), and the simulation results are as shown in Figure 2-11. 


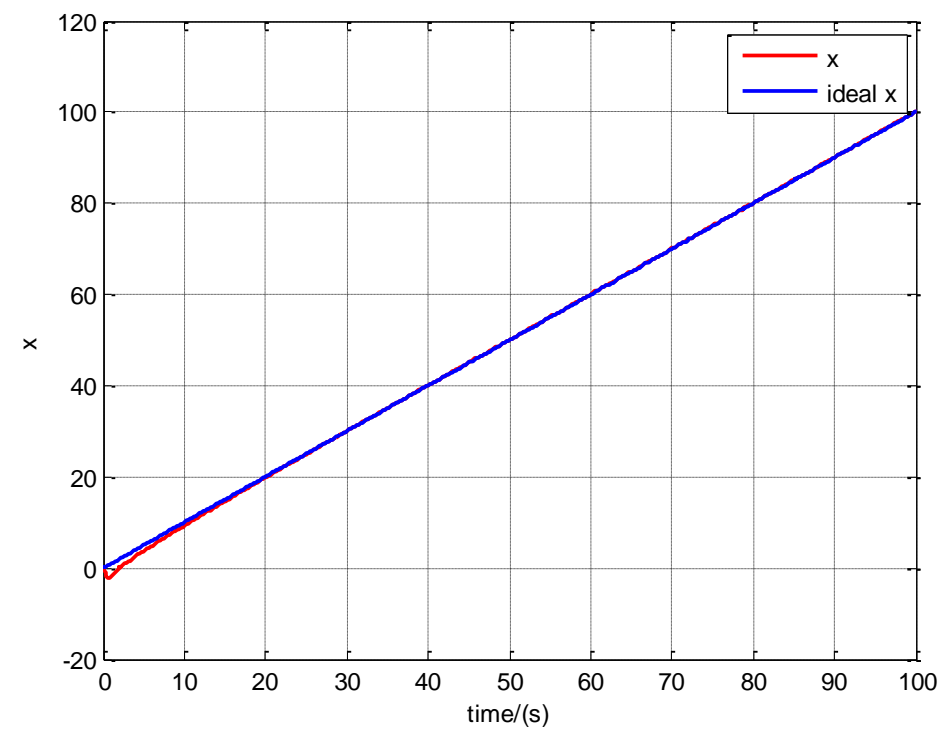

Figure 2. Tracking Curve of Aircraft Centroid Location $x_{1}(t)$



Figure 3. Tracking Error Curve of Aircraft Centroid Location $x_{1}(t)$ 




Figure 4. Tracking Curve of Aircraft Centroid Location $y_{1}(t)$

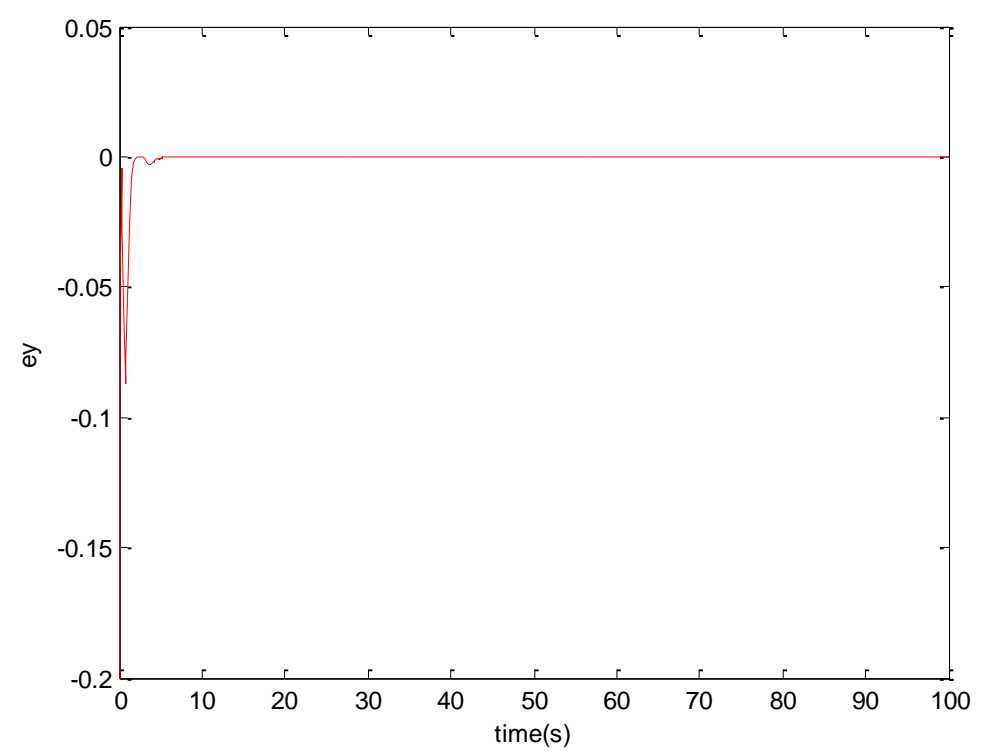

Figure 5. Tracking Error Curve of Aircraft Centroid Location $y_{1}(t)$ 


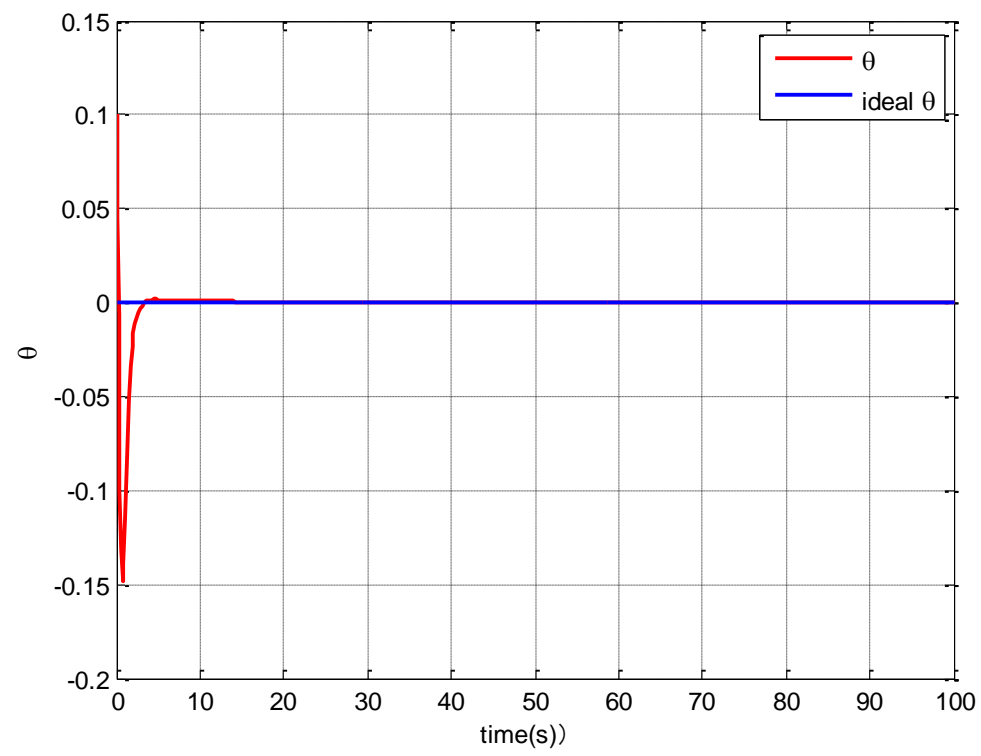

Figure 6. Tracking Curve of Aircraft Rolling Angle $\theta(t)$

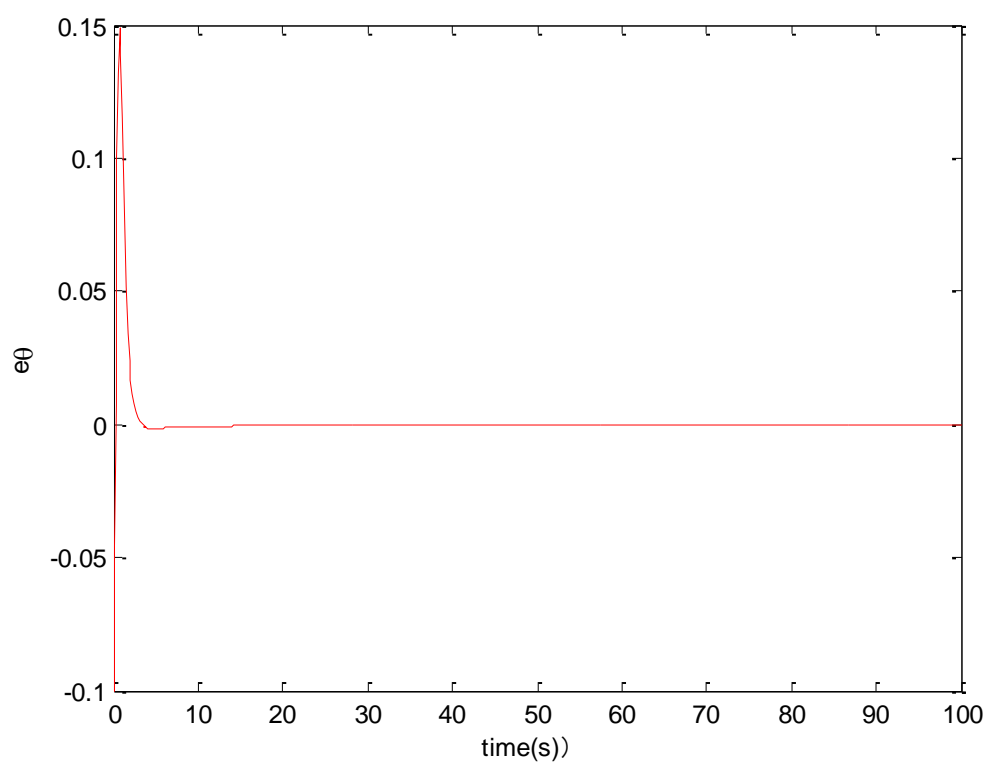

Figure 7. Tracking Error Curve of Aircraft Rolling Angle $\theta(t)$

As can be seen from Figure 2-7, aircraft centroid location $x_{1}(t), y_{1}(t)$ and rolling angle $\theta(t)$ can quickly track the desired set point, and the final tracking error is 0 . 


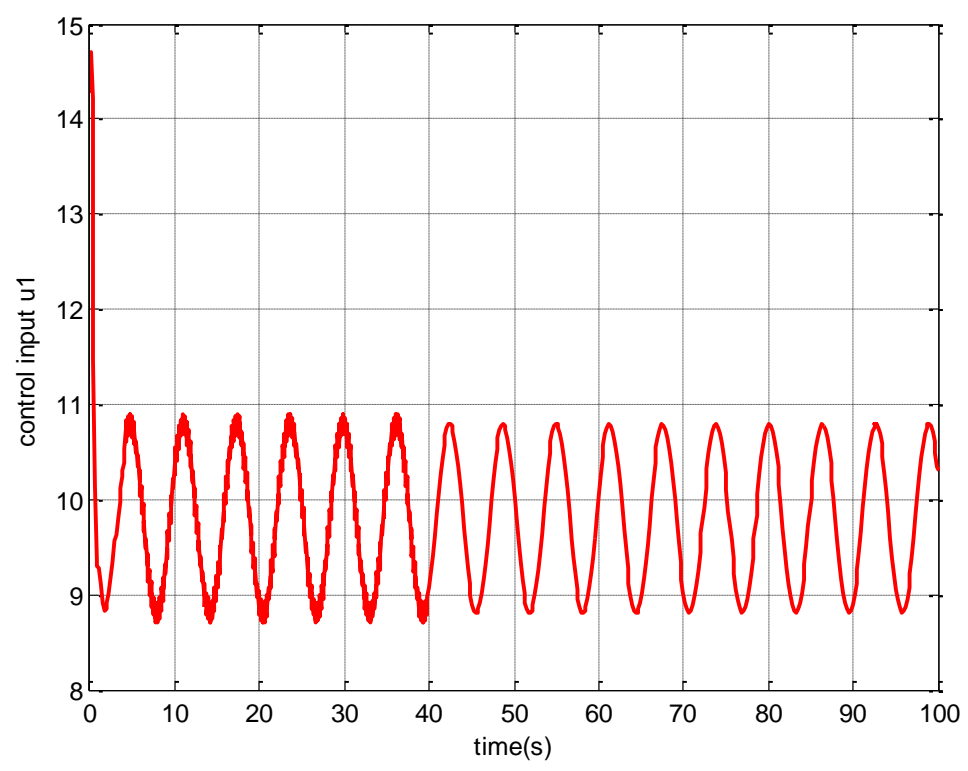

Figure 8. Aircraft Bottom Drive Control Input $u_{1}(t)$



Figure 9. Aircraft Rolling Control Input $u_{2}(t)$ 


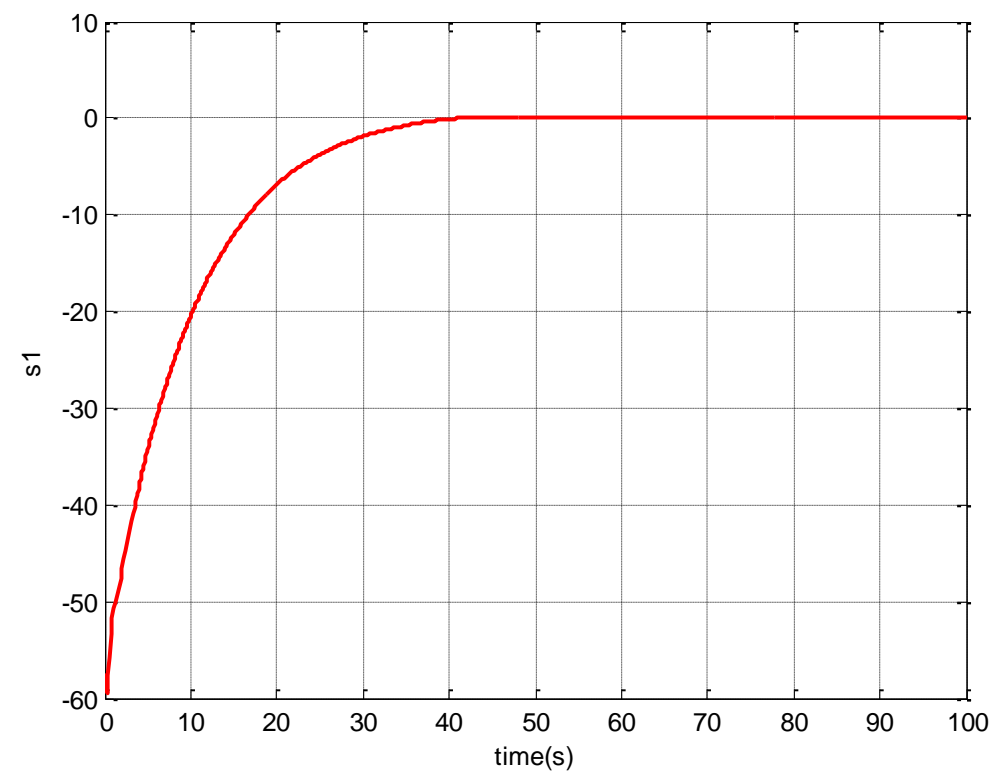

Figure 10 The Sliding Mode Surface $s_{1}(t)$ of Aircraft

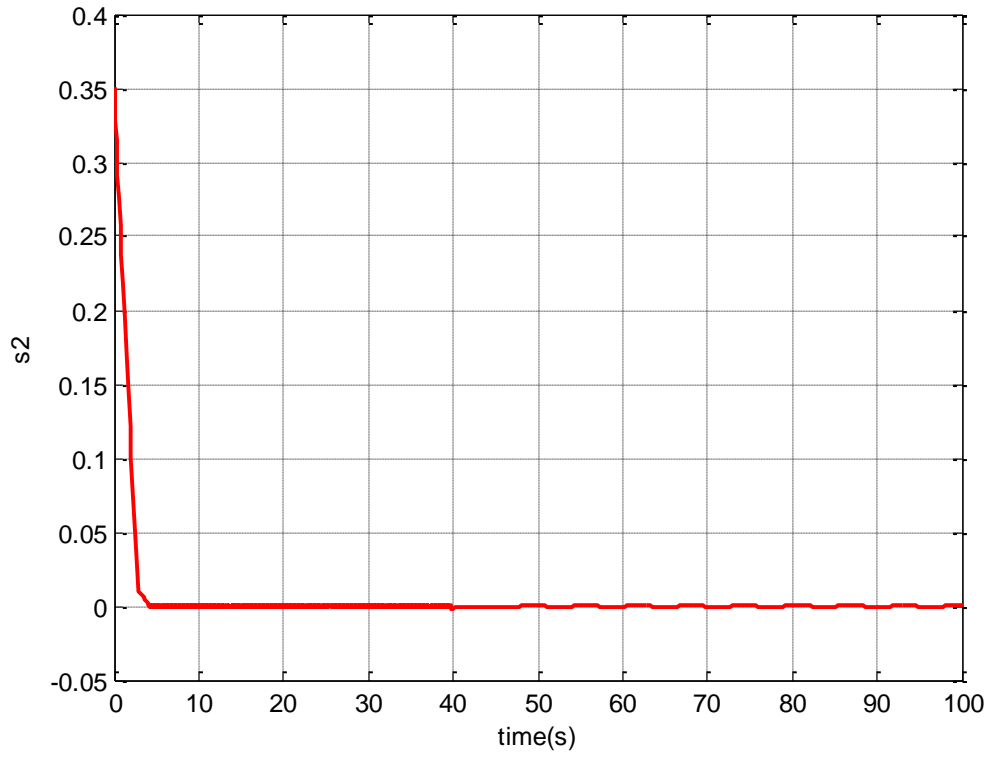

Figure 11 The Sliding Mode Surface $s_{2}(t)$ of Aircraft

Figure 8 and 9 indicate that the output buffeting in figure 8 is great in the previous $40 \mathrm{~s}$ which is due to the fact that the sliding mode surface $s_{1}(t)$ in figure 10 reaches the sliding mode surface of $s_{1}(t)=0$ at $40 \mathrm{~s}$. The output buffeting in Figure 9 has very short duration because the sliding mode surface $s_{2}(t)$ in figure 11 of aircraft will soon reach the sliding mode surface of $s_{2}(t)=0$. 


\section{Conclusion}

This paper studies the trajectory tracking of under-actuated VTOL aircraft with three degrees of freedom and two control inputs under various coupling effects. By the input and coordinate transformation, the dynamic equation of the system is transformed into decoupled standard under-actuated form and the sliding mode controller is designed. Then Lyapunov stability theorem is used to derive sliding mode control law which could ensure that the system asymptotically converges to the given trajectory. The simulation has demonstrated the effectiveness of this method.

\section{References}

[1] G. Wang and $\mathrm{Z}$. Wu, "Overall scheme analysis and control strategy of vertical take off and landing unmanned aerial vehicle",Aircraft Design, vol. 92, no. 3,(2006),pp.25-30.

[2] I. Kolmanovsky and N H. McClamroch, "Developments in nonholonomic control problems", Control Systems, IEEE, vol. 15, no. 6,(1995),pp.20-36.

[3] Y. Orlov, L. T. Aguilar and L. Acho, "Model orbit robust stabilization (MORS) of Pendubot with application to swing up control", Decision and Control,2005 and 2005 European Control Conference. CDC-ECC'05. 44th IEEE Conference on. IEEE, (2005).October 15-19.

[4] Z. Lv, A. Halawani, and S. Feng, "Multimodal hand and foot gesture interaction for handheld devices", ACM Transactions on Multimedia Computing, Communications, and Applications, vol. 11, no. 1s, (2014), pp.1-10.

[5] Q. Lin, Q. Zhu, and P. Huang, "A novel hybrid multi-objective immune algorithm with adaptive differential evolution”, Computers \& Operations Research,vol. 11, no. 7, (2015), pp.95-111.

[6] J. Chen, Q. Lin, and Q. Hu, “Application of novel clonal algorithm in multi-objective optimization”, vol. 19, no. 2, (2010), pp.239-266.

[7] W. Wang, J. Yi, and D. Zhao, "Design of a stable sliding-mode controller for a class of second-order underactuated systems", vol. 151, no. 6, (2004), pp. 683-690.

[8] Y. Zhao,"Robust Predictive Control of Input Constraints and Interference Suppression for Semi-Trailer System", International Journal of Control and Automation, vol.7, no.7, (2014), pp. 371-382.

[9] X. Wang, J. Liu and K. Y. Cai, "Tracking control for a velocity-sensorless VTOL aircraft with delayed outputs", Automatica, vol.45, no.11, (2009), pp. 2876-2882.

[10] R. Olfati-Saber, "Global configuration stabilization for the VTOL aircraft with strong input coupling", Automatic Control, IEEE Transactions on, vol.47, no.10, (2002), pp.1949-1952.

[11] R. Xu and $\square$ Ozg $\square$ uner $\square$ U.,"Sliding mode control of a class of underactuated systems", Automatica, vol.44, no.1, (2008), pp.233-241.

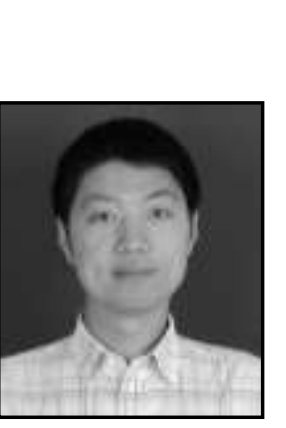

\section{Author}

Yang Zhao, received the $\mathrm{PhD}$ degree from Jilin University in 2015.Now he serves as a teacher at Department of Mechanical and Electrical Engineering, Guangdong University of Science \& Technology. His research interests include Optimization algorithm, Predictive control. 